\title{
Genetics of $\alpha$-amanitin resistance in a natural population of Drosophila melanogaster
}

\author{
DAVID J. BEGUN* \& PENN WHITLEY \\ Section of Integrative Biology and Institute for Cellular \& Molecular Biology, University of Texas, \\ Patterson Labs, C0930, Austin, TX 78712, U.S.A.
}

\begin{abstract}
The genetic basis of variation in resistance to natural toxins is of interest for both ecological and evolutionary genetics. The wide variety of larval resources used by Drosophila, both within and between species, makes flies an excellent system for studying causes and consequences of selection resulting from exposure to natural toxins associated with different resources. In this study we carry out a genetic analysis of $\alpha$-amanitin resistance in a population sample of Drosophila melanogaster. Data from mapping crosses of chromosome III support a role for a naturally occurring polymorphism in a multidrug resistance gene $(M d r 65 A)$ in $\alpha$-amanitin resistance. However, there are no amino acid differences between resistant and sensitive chromosomes at $M d r 65 A$. Therefore, if $M d r 65 A$ mutants contribute to the difference between $\alpha$-amanitin-resistant and $\alpha$-amanitin-sensitive third chromosome lines, the underlying cause is a gene regulatory mutation.
\end{abstract}

Keywords: evolution, Mdr65A, multidrug resistance, P-glycoprotein, population, selection.

\section{Introduction}

Our understanding of the genetics of naturally occurring intraspecific phenotypic variation is still simplistic. Most of our data come from systems in which there is a dramatic morphological or biochemical phenotype, and in which the genetics are fairly simple, and therefore, amenable to classical genetic analysis (e.g. single-locus pigmentation polymorphisms in animals and plants, insecticide resistance, herbicide resistance). It is unclear whether we should draw generalizations about the genetics of phenotypic variation from these examples. In Drosophila melanogaster, we know of at least two cases besides insecticide resistance (Ffrench-Constant et al., 1993) in which natural phenotypic variation has a relatively simple genetic basis. The two phenotypes are the rover/sitter behavioural polymorphism which is caused by variation at a protein kinase locus (Osborne et al., 1997), and resistance to parasitoid wasps, which also has a simple genetic basis (Orr \& Irving, 1997). In many cases, however, classical genetic analysis of interesting phenotypic variation might not provide much information about the genetics once the most simple single-locus two-allele model is rejected. This is because several genetic models may be compatible with data from systems in which the underlying genetics are

*Correspondence. E-mail: djbegun@mail.utexas.edu only marginally more complex than the single-locus two-allele case. The introduction of QTL mapping to evolutionary genetics should eventually help rectify this situation. For example, bristle number in D. melanogaster, a 'classic' quantitative or continuous trait, has yielded to QTL analyses that revealed the presence of alleles of major effect at candidate bristle mutant genes (Lai et al., 1994; Long et al., 1995, 1996). The research programme on the genetics of bristle variation points out a strong point of $D$. melanogaster as a model system. If one's goal is to describe the genetic properties of alleles at particular genes, then one must use an experimental system such as D. melanogaster, in which sophisticated genetic approaches are available.

Drosophila exist on a wide variety of substrates including rotting fruit or vegetation, fungus, cactus, flowers, and tree sap (reviewed in Powell, 1997). This raises the general question of the nature of segregating variation that might permit Drosophila populations to evolve the capacity to use resources containing novel plant, bacterial or fungus-derived natural toxins, and the connection of such variation to divergence in resource use during Drosophila evolution. A surprising result from previous analyses of a sample of $D$. melanogaster isofemale lines was the presence of some lines that were genetically resistant to $\alpha$-amanitin (Phillips et al., 1982). $\alpha$-amanitin is found in the mushrooms used as a resource by some species in the 'quinaria' group of Drosophila (Spicer \& Jaenike, 1996). However, given the fact that 
D. melanogaster lives primarily on fruit, there is no reason to think this species has a history of exposure to this compound. The genetic basis of resistance in the aforementioned $D$. melanogaster isofemale lines was fairly simple. Most of the variation was attributable to chromosome III, and although the data did not fit a single-locus two-allele model, a two-locus model with resistance alleles dominant to sensitive alleles was compatible with the data. Using such a genetic model, one gene contributing to resistance was mapped to each arm of chromosome III.

The toxic effect of $\alpha$-amanitin results from its interference with proper functioning of RNA polymerase II. Studies of resistant lines of D. melanogaster, however, have provided no evidence that their resistance was the result of variation in RNA polymerase II function (Phillips et al., 1982), even though evolved $\alpha$-amanitin resistance in the laboratory in both $D$. melanogaster and Caenorhabditis elegans can be caused by mutations at RNA polymerase II (Greenleaf et al., 1979; Sanford et al., 1983). Therefore, the genetic basis of variation for resistance to $\alpha$-amanitin as well as its evolutionary and ecological significance in $D$. melanogaster remains a mystery.

Here we present a genetic analysis of $\alpha$-amanitin resistance among recently isolated isogenic chromosome III lines. We also examine the possible contribution of variation at $M d r 65 A$, a multidrug resistance gene located on chromosome III, to variation in $\alpha$-amanitin resistance. Clearly, drug resistance is an incidental consequence of natural selection for other functions. One such function could be protection from naturally occurring environmental toxins, although functions relating to transport of endogenous molecules have also been proposed (Borst \& Schinkel, 1996, 1997). Despite the attention given to these proteins by virtue of their medical importance, the ecological and evolutionary significance of variation in $M d r$ genes within and between species remains unexplored.

\section{Materials and methods}

\section{Population samples}

Isofemale lines were established from females captured at the Wolfskill Orchard in Winters, CA, in summer 1995. Individual homozygous chromosome III lines were made through crosses of flies from these isofemale lines to appropriate balancer stocks.

\section{$\alpha$-amanitin-containing food}

For the survey of $\alpha$-amanitin resistance among chromosome III lines, $\alpha$-amanitin (Sigma A2263) suspended in distilled water to a final concentration of $1 \mathrm{mg} \mathrm{mL}^{-1}$ was added to standard cornmeal-molasses-agar Drosophila food prior to the food being poured into vials. The final concentration of $\alpha$-amanitin was about $1 \mu \mathrm{g} \mathrm{mL}^{-1}$ (vials contain about $7 \mathrm{~mL}$ of food). For segregation analyses of resistant and sensitive lines, $\alpha$-amanitin was added to vials that had already been poured. In these experiments vials were microwaved until the food was liquefied. Seven $\mu \mathrm{L}$ of $\alpha$-amanitin from a $1 \mathrm{mg} \mathrm{mL}^{-1}$ stock in water was then added to each vial. The food was then well-mixed with a small spatula and allowed to cool to room temperature. Preliminary experiments (not shown) have suggested that phenotypes of isogenic stocks were similar regardless of the mode of preparation of the $\alpha$-amanitincontaining food.

\section{$\alpha$-amanitin resistance of chromosome III lines}

Large numbers of adults from individual homozygous lines grown in bottles were collected and allowed to oviposit in Petri dishes containing a thin layer of Drosophila medium and a drop of yeast paste. Firstinstar larvae were then picked and gently placed onto the surface of vials containing food with $1 \mu \mathrm{g} \mathrm{mL}^{-1}$ $\alpha$-amanitin. Four replicate vials, each containing 30 first-instar larvae, were established for each isogenic line. Four control vials containing no $\alpha$-amanitin were established in the same way for each line. Vials containing first-instar larvae were incubated at $25^{\circ} \mathrm{C}$ for 14 days, at which time adults were counted. Resistance for each line was estimated as the proportion of flies eclosing from $\alpha$-amanitin-containing vials by Day 14 , divided by the proportion of flies eclosing from control vials by Day 14 . The value of $\alpha$-amanitin resistance for each line was the average resistance over the four replicate vials.

\section{Genetic analysis of extreme chromosome III lines}

Two lines, one sensitive (III-27) and one resistant (III-25), identified in the survey described above were used in a segregation analysis. Both lines had excellent viability in control vials [mean (SE) viabilities for III-27 and III-25 were $0.933(0.024)$ and 0.925 (0.032), respectively]. Sensitive III-27 females were crossed to III-25 resistant males to generate heterozygous F1 females. These F1 females were backcrossed to sensitive III-27 males (10 pairs per vial) and allowed to oviposit for three days on food to which $\alpha$-amanitin had been added at a concentration of $1 \mu \mathrm{g} \mathrm{mL} L^{-1}$. The adults were then discarded. Vials were incubated at $25^{\circ} \mathrm{C}$. Control crosses were carried out in the same way except that $\alpha$-amanitin was not added to the food. Adults were collected on Day 
14 and were stored individually in $1.5 \mathrm{~mL}$ microcentrifuge tubes at $-20^{\circ} \mathrm{C}$. DNA preps from single flies were made using a scaled-down version of the Bender et al. (1983) protocol.

The following chromosome III microsatellite markers described in Schug et al. (1998) (cytological locations in parentheses) were informative in our cross of lines III-27 and III-25: rhoB (62A), ect (67D8), $17 c d c 2 z$ (84), ula (93C), and mlc (98). PCR primers for these microsatellites were end-labelled with ${ }^{33} \mathrm{P}$ and used to amplify microsatellites from single flies. The resulting products were run on $5 \%$ polyacrylamide gels that were then dried and exposed to film to generate autoradiograms. We also scored a $T a q \mathrm{I}$ restriction site difference between lines III-27 and III-25 at the Mdr65 A locus (65) on 1.5\% ethidium bromide-stained agarose gels.

\section{Sequencing}

DNA sequencing of lines III-25 and III-27 was accomplished through a combination of direct sequencing of PCR products, sequencing of genomic clones, or sequencing of clones derived from PCR products amplified with a high-fidelity polymerase. Sequences were run on an ABI 377 automated sequencer. Genbank accession numbers are AF251286 and AF251287.

\section{Results}

\section{Distribution of $\alpha$-amanitin resistance}

Figure 1 shows the mean $\alpha$-amanitin resistance of 20 homozygous chromosome III lines isolated from flies collected in a California orchard during the summer of 1995. Differences among lines in mean $\alpha$-amanitin resistance were highly significant by ANOVA $(\mathrm{SS}=6.00$, $\mathrm{MS}=0.316, F=10.24, P<0.0001$, d.f.=19). Many lines were fairly resistant at an $\alpha$-amanitin concentration

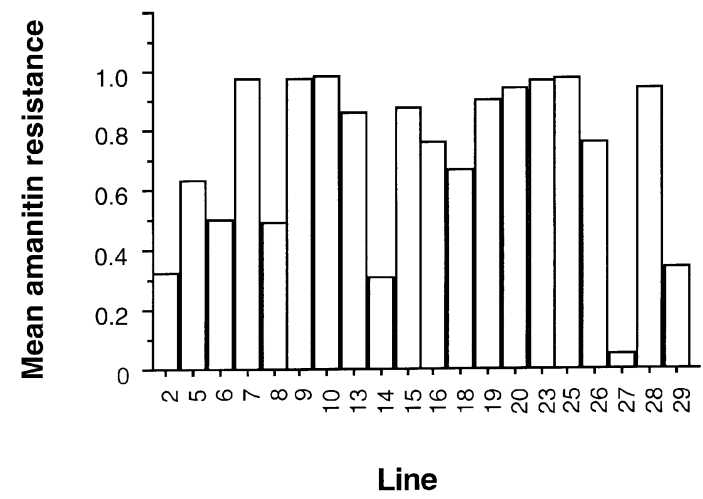

Fig. 1 Line means for $\alpha$-amanitin resistance among 20 isogenic chromosome III lines of D. melanogaster. of $1 \mu \mathrm{g} \mathrm{mL}^{-1}$. However, a few lines were sensitive at this concentration; one line, III-27, was very sensitive to $1 \mu \mathrm{g} \mathrm{mL}^{-1} \alpha$-amanitin. The senstivity of line III-27 was highly repeatable in subsequent replicates (data not shown).

\section{Analysis of a candidate locus}

Previous mapping data (Phillips et al., 1982) indicated the presence of a gene contributing to $\alpha$-amanitin resistance at about genetic map position 19 on chromosome III. Therefore, we inspected this region of the genome for candidate resistance genes. One candidate locus, Mdr65 A, is located at polytene band 65A (Wu et al., 1991), which corresponds to a genetic map position of about 20. Given the earlier mapping data and the known function of $M d r$ genes in resistance to cytotoxic compounds, we considered $M d r 65 A$ to be a promising candidate locus for $\alpha$-amanitin resistance. We used PCR to amplify fragments of the $M d r 65 A$ locus in lines III-27 and III-25. We then digested these PCR products with several four-cutter restriction enzymes, and ran the resulting digests on $1.5 \%$ agarose gels. An easily scored TaqI presence/absence polymorphism that distinguished the Mdr65 A alleles of lines III-27 and III-25 was used in a segregation test.

Heterozygous III-27/III-25 females were backcrossed to sensitive homozygous III-27 males and allowed to oviposit on experimental or control food. If there were no heterozygous effect of the $M d r 65 A$ locus or a linked site on survival in the presence of $\alpha$-amanitin, then we would expect the $M d r 65 A$ genotype ratios in experimental and control crosses to be the same. Alternatively, if there was a heterozygous effect of $M d r 65 A$ or a linked site on $\alpha$-amanitin resistance, then we would expect to see an excess of heterozygous genotypes among the survivors, compared to the proportion of heterozygous genotypes among the control progeny.

The results of the segregation test of $M d r 65 A$ alleles in experimental and control crosses are shown in Table 1.

Table 1 Association of an RFLP marker at Mdr65A with survival in the presence of $\alpha$-amanitin

\begin{tabular}{lcc}
\hline & III-27/III-27 & III-25/III-27 \\
\hline Experimental & 34 & 181 \\
Control & 80 & 108 \\
\hline
\end{tabular}

III-27/III-25 females were crossed to III-27/III-27 males in the presence (experimental) and absence (control) of $1 \mu \mathrm{g} / \mathrm{mL}$ $\alpha$-amanitin. III-25 is resistant; III-27 is sensitive. Surviving progeny were scored for a TaqI/Mdr65 A polymorphism. The null hypothesis that there is no effect of $M d r 65 A$ or of a linked marker on $\alpha$-amanitin resistance predicts equal numbers of progeny in the two genotypic classes in experimental and control crosses. 
Genotype ratios in the control cross were not significantly different $\left(\chi^{2}=2.10\right)$ from the expected Mendelian ratios of 1:1. However, a binomial test revealed a marginally significant excess of heterozygotes in the control cross ( $P=0.04$, two-tailed). This result is probably explained by inbreeding depression. There was a very large excess of heterozygotes in the experimental cross. Genotype ratios in the experimental cross were highly significantly different from the expected 1:1 ratio $\left(\chi^{2}=56.8, P<10^{-5}\right)$ and from the ratio in the control cross $\left(\chi^{2}=35.4 ; P<10^{-5}\right)$. Therefore, even with a small degree of inbreeding depression, we can conclude confidently that there is a very large heterozygous effect of the $M d r 65 A$ allele or of a tightly linked allele at another locus on the probability that a zygote produced in our experimental cross would survive to adulthood when exposed to $1 \mu \mathrm{g} \mathrm{mL}^{-1} \alpha$-amanitin. The magnitude of the effect is surprising given that the genetic backgrounds of stocks were not controlled.

\section{Marker analysis of chromosome III}

Replicates of cross of III-27/III-25 females $\times$ III-27/III27 males were established as described above. These females were allowed to oviposit on $1 \mu \mathrm{g} \mathrm{mL}^{-1} \alpha$-amanitin food. Adults eclosing by Day 14 were genotyped at the $M d r 65 A$ locus as well as at 5 microsatellite loci distributed across the third chromosome. The resulting genotype ratios (heterozygote: heterozygote + homozygote) among 100 survivors are shown in Fig. 2. In each case the expected genotype ratio if there were no effect of the marker on survival is $50 \%$. As was observed in the previous experiment, the progeny genotype ratio at $M d r 65 A$ was extremely skewed towards an excess of heterozygotes. There was also weaker evidence for an association of the microsatellite marker at cytological position 98 with survival in the presence of $\alpha$-amanitin. Remaining marker genotype ratios were close to the expected 1:1 ratio. The fact that the proportion of heterozygotes at the remaining markers was slightly, but consistently, greater than $50 \%$ probably results from mildly deleterious recessive alleles that cause slight inbreeding depression. Importantly, genotype ratios of flanking markers on both sides of $M d r 65 A$ show little deviation from Mendelian expectations. This observation strengthens considerably the notion that an allele at or very closely linked to $M d r 65 \mathrm{~A}$ has a significant heterozygous effect on survival in the presence of $\alpha$-amanitin. The results also suggest the presence of variation at a second locus near the tip of $3 \mathrm{R}$ that can affect survival in the presence of $\alpha$-amanitin. This observation is consistent with the results from earlier experiments (Phillips et al., 1982) indicating the existence of such an allele at genetic map position 100 of chromosome III.
Fig. 2 Proportion of heterozygotes among survivors ( $\mathrm{Y}$-axis) vs. the physical location of the marker along the third chromosome of D. melanogaster (X-axis).

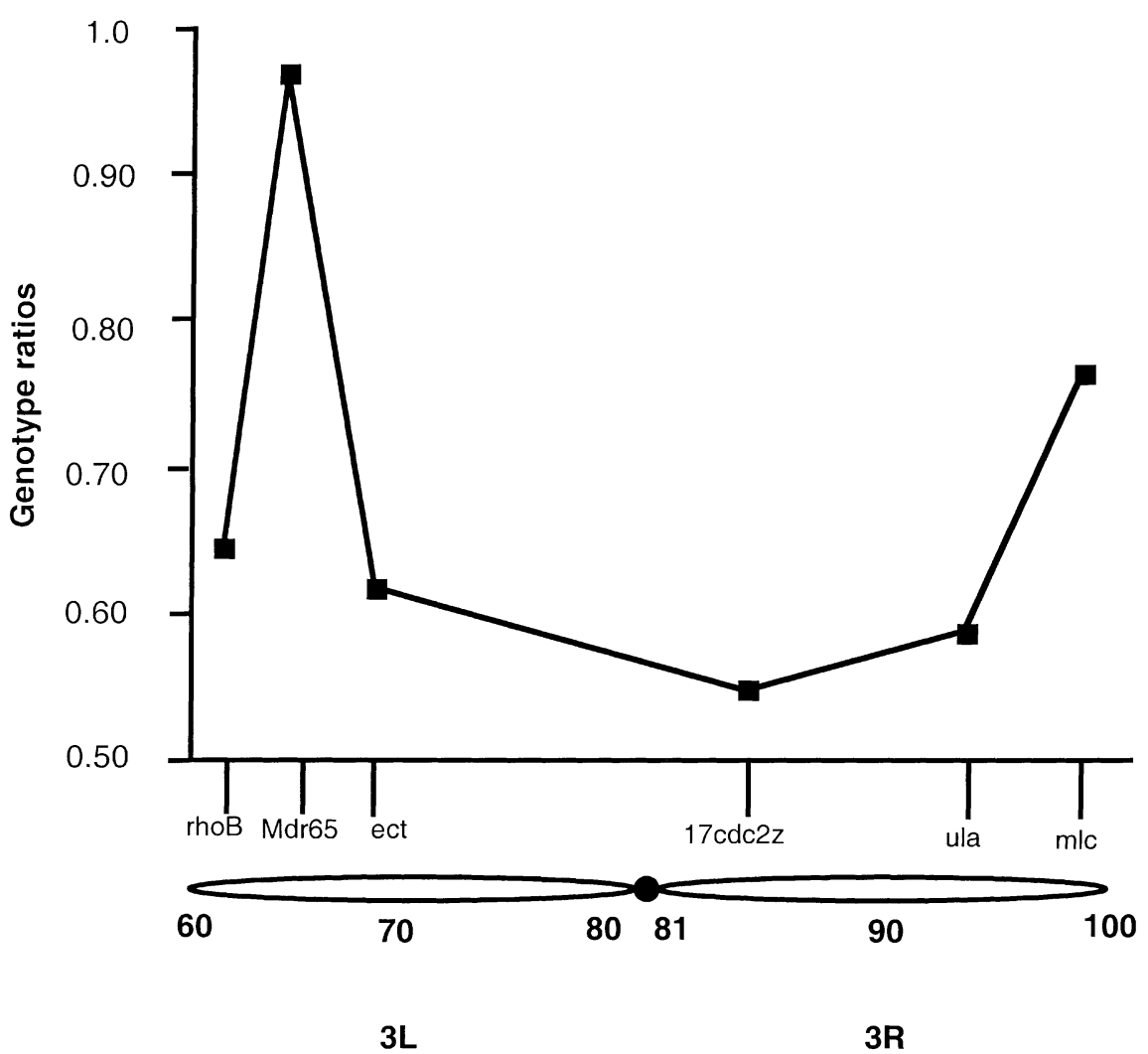

(C) The Genetical Society of Great Britain, Heredity, 85, 184-190. 


\section{DNA polymorphisms at Mdr65A}

We sequenced the $M d r 65 A$ region of lines III-27 and III-25. The sequenced region of $7331 \mathrm{bp}$ includes $1746 \mathrm{bp} 5^{\prime}$ of the initiation codon, the entire proteincoding region, and $872 \mathrm{bp}$ of $3^{\prime}$ of the termination codon. The $5^{\prime}$-flanking region includes an untranslated exon. Locations of introns and exons were determined by comparison of our sequence and that of the Berkeley Drosophila Genome Project with the published cDNA (Wu et al., 1991). There are no amino acid differences between the Mdr65A alleles of lines III-27 and III-25. The numbers of $5^{\prime}$-flanking, silent-site polymorphisms, intron polymorphisms and $3^{\prime}$-flanking polymorphisms were 3, 7, 10 and 0 , respectively. The estimate of $\theta$ per silent site (Watterson, 1975) at Mdr65A is 0.004 . This is low given the position of this locus in a region of normal recombination (Begun \& Aquadro, 1992; Aquadro et al., 1994; Moriyama \& Powell, 1996), although the estimate, which is based on a sample size of only two alleles, should be considered rather crude. Given the absence of amino acid differences between alleles, if Mdr65A contributes to the phenotypic difference between lines III-27 and III-25, the cause must be a polymorphism or polymorphisms in the flanking regions or introns. The paucity of information on regulation of $M d r 65 A$ precludes speculation on the potential functional significance of the observed polymorphisms.

\section{Discussion}

We found very large phenotypic differences in $\alpha$-amanitin resistance among lines homozygous for recently isolated third chromosomes from a California population of Drosophila melanogaster. Genetic analysis of the phenotypic differences between a pair of extreme lines from this sample revealed strong evidence for an allele with a large heterozygous effect on resistance at or near the $M d r 65 A$ locus at cytological position 65, and weaker evidence for a resistance allele at a second locus near the tip of $3 \mathrm{R}$. These results are consistent with previous genetic results obtained through mapping of a resistant chromosome III from an isofemale line of unknown history against a laboratory chromosome marked with a number of recessive, visible mutants (Phillips et al., 1982).

Multidrug resistance genes (also referred to as P-glycoproteins) are members of the ABC-transporter gene family (Higgins, 1992). MDRs are large (c. 1300 amino acids long) transmembrane proteins that function in drug efflux through either a pump or 'flippase' mechanism (Gottesman et al., 1995). The biology of $M d r$ proteins is of great interest as they are often implicated in the development of chemotherapy-resistant tumours in humans (e.g. Germann, 1996). These genes were first discovered by virtue of this phenotype, which probably results from the role of MDR in efflux of cytotoxic chemotherapeutic compounds from cells (Gottesman et al., 1995). Our genetic data, considered alongside the function of $M d r$ genes, certainly makes $M d r 65 A$ a candidate locus for $\alpha$ amanitin resistance. DNA sequence analysis of $M d r 65 A$ from a resistant and sensitive strain revealed no amino acid differences between the sensitive and resistant chromosomes. However, there are a number of intron and 5 '-flanking differences at $M d r 65 A$ between our sensitive (III-27) and resistant (III-25) chromosomes. Thus, if $M d r 65 A$ alleles contribute to phenotypic variation for $\alpha$ amanitin resistance, the underlying cause is a difference in regulation rather than in amino acid sequence. The dominant effect of the $M d r 65 \mathrm{~A}$ marker on survival is certainly consistent with a regulatory effect. For example, dominant insecticide resistance in mosquitoes can either be due to upregulation or duplication of a locus (Pasteur \& Raymond, 1996). Similarly, dominant, laboratoryinduced mutations caused by inversions in Drosophila are often thought to be the result of chromosome rearrangements that cause inappropriate regulation of genes near inversion breakpoints. Multidrug resistance in mammalian cell lines often results from amplification of $M d r$ genes (reviewed Gottesman et al., 1995), establishing the importance of dosage effects of $M d r$ genes on the resistance phenotype.

It is extraordinarily difficult to use results from sequence analysis to prove that a particular mutation or group of mutations in a particular gene is the cause of phenotypic variation. We are fortunate, however, in that the phenotypic difference between lines is very large, and is explained almost entirely by a single chromosomal region. If variation in $M d r 65 A$ is the cause of the phenotypic difference between lines, we should be able to 'rescue' the conditional lethal phenotype of a sensitive strain with an Mdr65A transgene from line III-25.

We have weaker evidence for a gene on the tip of $3 \mathrm{R}$ affecting resistance to $\alpha$-amanitin. Are there any candidate resistance genes in this region of the D. melanogaster genome? Protein kinase C98E (Pkc98E) is located at polytene band $98 \mathrm{~F}$. PKC has several roles, one of which is phosphorylation of $M d r$ proteins (Chambers et al., 1990). Thus, to the extent that $M d r 65 A$ is a candidate locus for $\alpha$-amanitin resistance, the genetic data suggest that $P k c 98 F$ could reasonably be considered a candidate gene. However, this speculative hypothesis is weakened by results from mammalian cells that call into question the importance of phosphorylation in mediating P-glycoprotein activity 
(Germann et al., 1996; Goodfellow et al., 1996). The foraging locus codes for a protein kinase, and harbours a natural, two-allele behavioural polymorphism (Osborne et al., 1997). Thus, the presence of naturally occurring alleles of large effect in protein kinases is at least plausible.

We are still left with the mystery of why D. melanogaster populations harbour alleles of major effect for resistance to a toxin to which they have no apparent history of exposure. One possibility is that the genetic variation we observe has no fitness effects in nature. This seems unlikely, however, given the extremely large phenotypic differences among chromosomes in our experiments. A more likely explanation is that $\alpha$-amanitin resistance is a pleiotropic effect of genetic variation maintained by selection on some other phenotype (e.g. resistance to natural toxins to which D. melanogaster is exposed in nature). Determination of the ecological and evolutionary significance of the genetic variation we have observed will require identification of the specific molecular changes that cause the phenotypic variation, the histories and current allele frequencies of such mutants, and descriptions of their interactions with other genotypes and other compounds.

\section{Acknowledgements}

This work was supported by NIH GM55298.

\section{References}

AQUADRO, C. F., BEGUN, D. J. AND KINDAHL, E. C. 1994. Selection, recombination, and DNA polymorphism in Drosophila. In: Golding, B. (ed.) Non-Neutral Evolution: Theories and Molecular Data, pp. 46-56. Chapman \& Hall, New York.

BEGUN, D. J. AND AQUADRO, C. F. 1992. Levels of naturally occurring DNA polymorphism correlate with recombination rates in D. melanogaster. Nature, 356, 519-520.

BENDER, W., SPIERER, P. AND HOGNESS, D. S. 1983. Chromosomal walking and jumping to isolate DNA from the Ace and rosy loci and the Bithorax complex in Drosophila melanogaster. J. Molec. Biol., 168, 17-33.

BORST, P. AND SCHINKEL, A. H. 1996. What have we learnt thus far from mice with disrupted P-glycoprotein genes? Eur. J. Cancer Biol., 32A (6), 985-990.

BORST, P. AND SCHINKEL, A. H. 1997. Genetic dissection of the function of mammalian P-glycoproteins. Trends Genet., 13, 217-222.

CHAMBERS, T. C., MCAVOY, E. M., JACOBS, J. W. AND EILON, G. 1990. Protein kinase C phosphorylates P-glycoprotein in multidrug resistant human $\mathrm{KB}$ carcinoma cells. J. Biol. Chem., 265, 7679-7686.

FFRENCH-CONSTANT, R. H., ROCHELEAU, T., STEICHEN, J. C. AND CHALMERS, A. E. 1993. A point mutation in a Drosophila
GABA receptor confers insecticide resistance. Nature, 363, 449-451.

GERMANN, U. A. 1996. P-glycoprotein - A mediator of multidrug resistance in tumour cells. Eur. J. Cancer Biol., 32A (6), 927-944.

GermanN, U. A., CHAMbers, T. C., AMbudKAR, S. V., LiCht, T., CARDARELli, C. O., PASTAN, I. ET AL. 1996. Characterization of phosphorylation-defective mutants of human P-glycoprotein expressed in mammalian cells. J. Biol. Chem., 271, 1708-1716.

GOODFEllow, H. R., SARdini, A., RUETZ, S., CAllaghan, R., Gros, P., MCNAughton, P. A. ET AL. 1996. Protein kinase C-mediated phosphorylation does not regulate drug transport by the human multidrug resistance P-glycoprotein. J. Biol. Chem., 271, 13668-13674.

GOTTESMAN, M. M., HRYCYNA, C. A., SCHOENLEIN, P. V., GERMANN, U. A. AND PASTAN, I. 1995. Genetic analysis of the multidrug transporter. Ann. Rev. Genet., 29, 607-649.

GREENLEAF, A. L., WEEKS, J. R., VOELKER, R. A., OHNISHI, S. AND DICKSON, B. 1979. $\alpha$-Amanitin-resistant D. melanogaster with an altered RNA polymerase II. Cell, 18, 613-622.

HIGGINS, C. F. 1992. ABC Transporters: From Microorganisms to Man. Ann. Rev. Cell Dev. Biol., 8, 67-113.

LAI, C., LYMAN, R. F., LONG, A. D., LANGLEY, C. H. AND MACKAY, T. F. C. 1994. Naturally occurring variation in bristle number and DNA polymorphisms at the scabrous locus of Drosophila melanogaster. Science, 266, 1697-1702.

LONG, A. D., MULLANEY, S. L., REID, L. A., FRY, J. D., LANGLEY, C. H. AND MACKAY, T. F. C. 1995. High resolution mapping of genetic factors affecting abdominal bristle number in Drosophila melanogaster. Genetics, 139, 1273-1291.

LONG, A. D., MULLANEY, S. L., MACKAY, T. F. AND LANGLEY, C. H. 1996. Genetic interactions between naturally occurring alleles at quantitative trait loci and mutant alleles at candidate loci affecting bristle number in Drosophila melanogaster. Genetics, 144, 1497-1510.

MORIYAMA, E. AND POWELL, J. R. 1996. Intraspecific nuclear DNA variation in Drosophila. Mol. Biol. Evol., 13, 261-277.

ORR, H. A. AND IRVING, s. 1997. The genetics of adaptation: The genetic basis of resistance to wasp parasitism in Drosophila melanogaster. Evolution, 51, 1877-1885.

OSBORNE, K. A., ROBICHON, A., BURGESS, E., BUTLAND, S., SHAW, R. A., COUlThARD, A. ET $A L$. 1997. Natural behavior polymorphism due to a cGMP-dependent protein kinase of Drosophila. Science, 277, 834-836.

PASTEUR, N. AND RAYMOND, M. 1996. Insecticide resistance genes in mosquitoes: Their mutations, migration, and selection on field populations. J. Hered., 87, 444-449.

PHILliPS, J. P., WILlmS, J. AND PITT, A. 1982. $\alpha$-Amanitin resistance in three wild strains of Drosophila melanogaster. Can. J. Genet. Cytol., 24, 151-162.

POWEll, J. R. 1997. Progress and Prospects in Evolutionary Biology: the Drosophila Model. Oxford University Press, New York.

SANFORD, T., GOLOMB, M. AND BIDDLE, D. L. 1983. RNA polymerase II from wild-type and $\alpha$-amanitin-resistant strains of C. elegans. J. Biol. Chem., 258, 12804-12809. 
SCHUG, M. D., WETTERSTRAND, K. A., GAUDETTE, M. S., LIM, R. H., HUTTER, C. M. AND AQUADRO, C. F. 1998. The distribution and frequency of microsatellite loci in Drosophila melanogaster. Molec. Ecol., 7, 57-70.

SPICER, G. AND JAENIKE, J. 1996. Phylogenetic analysis of breeding site use and $\alpha$-amanitin tolerance within the Drosophila quinaria species group. Evolution, 50, 2328-2337.
WATTERSON, G. A. 1975. On the number of segregating sites in genetical models without recombination. Theor. Pop. Biol., 7, 256-276.

WU, C.-T., BUDDING, M., GRIFFIN, M. S. AND CROOP, J. M. 1991. Isolation and characterization of Drosophila multidrug resistance gene homologs. Mol. Cell. Biol., 11, 39403948 . 